\title{
INTEGRAL AVERAGES AND OSCILLATION CRITERIA FOR HALF-LINEAR PARTIAL DIFFERENTIAL EQUATION
}

\author{
ROBERT MAŘÍK \\ MENDEL UNIVERSITY, DEPARTMENT OF MATHEMATICS \\ ZEMĚDĚLSKÁ 3, 61300 BRNO, CZECH REPUBLIC \\ E-MAIL: MARIKOMENDELU.CZ
}

\begin{abstract}
The technique of weighted integral averages, known in the oscillation theory of ordinary differential equations, is extended to the half-linear partial differential equation

$$
\operatorname{div}\left(\|\nabla u\|^{p-2} \nabla u\right)+c(x)|u|^{p-2} u=0 . \quad p>1
$$

This technique is used to obtain new oscillation criteria for (E) on the unbounded domains.

Keywords. $p$-Laplacian, oscillatory solution, Riccati equation, partial differential equation, half-linear equation

AMS Class. 35J60, 35B05

Running head: Integral averages and PDE

\section{INTRODUCTION}

Consider the partial differential equation with $p$-Laplacian and the nonlinearity of the Emden-Fowler type

$$
\Delta_{p} u+c(x) \Phi(u)=0,
$$

where $\Delta_{p} u \equiv \operatorname{div}\left(\| \nabla u||^{p-2} \nabla u\right), p>1$ is the $p$-Laplacian, $\Phi(u)=|u|^{p-2} u=$ $|u|^{p-1} \operatorname{sgn} u, x=\left(x_{i}\right)_{i=1}^{n} \in \mathbb{R}^{n},\|\cdot\|$ is the usual Euclidean norm in $\mathbb{R}^{n}$ and $\nabla=\left(\frac{\partial}{\partial x_{i}}\right)_{i=1}^{n}$ is the usual nabla operator.

The equations with $p$-Laplacian have applications in various physical and biological problems - in the study of non-Newtonian fluids, in the glaciology and slow diffusion problems. For more detailed discussion about applications of equations with $p$-Laplacian the reader is referred to [2] and the references therein.

Among the equations with $p$-Laplacian equation (E) plays a special role. Since both terms $\Delta_{p} u$ and $\Phi(u)$ are homogeneous functions of the degree $p-1$, (E) has the so-called half-linear property: the constant multiple of every solution is also a solution of (E). From this reason some of the qualitative properties of half-linear equation (E) are similar to the properties of linear Schrödinger partial

\footnotetext{
${ }^{0}$ Supported by the Grant 201/01/0079 of the Czech Grant Agency
} 
differential equation

$$
\Delta u+c(x) u=0
$$

which can be obtained from (E) for $p=2$. Especially the Sturmian type theorems extends from (L) also to (E), see $[6,8]$.

Notation: $\Omega(a), \Omega(a, b), S(a), D$ and $D_{0}$ are the sets in $\mathbb{R}^{n}$ and $\mathbb{R} \times \mathbb{R}^{n}$ defined as follows:

$$
\begin{aligned}
\Omega(a) & =\left\{x \in \mathbb{R}^{n}: a \leq\|x\|\right\}, \\
\Omega(a, b) & =\left\{x \in \mathbb{R}^{n}: a \leq\|x\| \leq b\right\}, \\
S(a) & =\left\{x \in \mathbb{R}^{n}:\|x\|=a\right\}, \\
D & =\left\{(t, x) \in \mathbb{R} \times \mathbb{R}^{n}: t \geq\|x\| \geq t_{0}\right\}, \\
D_{0} & =\left\{(t, x) \in \mathbb{R} \times \mathbb{R}^{n}: t>\|x\| \geq t_{0}\right\},
\end{aligned}
$$

the number $q$ is a conjugate number to the number $p$, i.e., $q=\frac{p}{p-1},\langle\cdot, \cdot\rangle$ denotes the usual scalar product in $\mathbb{R}^{n}, \omega_{n}$ is the surface of the unit sphere in $\mathbb{R}^{n}$. Integration over the domain $\Omega(a, b)$ is performed introducing hyperspherical coordinates $(r, \theta)$, i.e.

$$
\int_{\Omega(a, b)} f(x) \mathrm{d} x=\int_{a}^{b} \int_{S(r)} f(x(r, \theta)) \mathrm{d} \mathrm{S} \mathrm{d} r
$$

where dS is the element of the surface of the sphere $S(r)$.

The function $c(x)$ is assumed to be locally Hölder continuous on $\varnothing\left(t_{0}\right)$. The solution of equation (E) is every function which satisfies (E) everywhere on $\varnothing\left(t_{0}\right)$.

The oscillation properties of equation (L) and its generalizations, which includes also the half-linear partial differential equation with $p$-Laplacian (E) has been extensively studied in the literature, see e.g. [1, 6, 7, 8, 14, 15, 17, 18, 19, 20, 22, 23]. The oscillation theory of (L) recognizes two types of oscillation. Equation (L) is said to be weakly oscillatory if every its solution has a zero outside of every ball in $\mathbb{R}^{n}$ and strongly oscillatory if every solution has a nodal domain outside of every ball in $\mathbb{R}^{n}$. Moss and Piepenbrick [16] showed that both definitions are equivalent if the function $c(x)$ is locally Hölder continuous. As far as the author knows, the possible equivalence between both types of oscillation remains an open question for (E). In the paper the first type of oscillation is used.

Definition 1.1. Let $\Omega$ be unbounded domain in $\mathbb{R}^{n}$. Equation (E) is said to be oscillatory in $\Omega$ if every its nontrivial solution defined on $\Omega \cap \Omega\left(t_{0}\right)$ has zero in $\Omega \cap \Omega(t)$ for every $t \geq t_{0}$. Equation (E) is said to be oscillatory, if it is oscillatory in $\mathbb{R}^{n}$.

The oscillation criteria are usually expressed in terms of integrals of the potential function $c(x)$ over the balls centered in the origin. Let us mention the following theorem, well-known in the area of second-order linear ordinary differential equations. 
Theorem A (Hartman-Wintner type oscillation criterion, [14]). Denote

$$
C(t)=\frac{p-1}{t^{p-1}} \int_{1}^{t} s^{p-2} \int_{\varnothing(1, s)}\|x\|^{1-n} c(x) \mathrm{d} x \mathrm{~d} s .
$$

If

$$
-\infty<\liminf _{t \rightarrow \infty} C(t)<\limsup _{t \rightarrow \infty} C(t) \leq \infty \quad \text { or if } \quad \lim _{t \rightarrow \infty} C(t)=\infty,
$$

then equation (E) is oscillatory.

Very general oscillation criterion which deduces oscillation of (E) from oscillation of ordinary half-linear differential equation is due to Jaroš, Kusano and Yoshida [8]. A variant of this theorem has been proved independently also by Došlý and Mařík [6].

Theorem B ( [6]). Let

$$
\tilde{c}(r)=\frac{1}{\omega_{n} r^{n-1}} \int_{S(r)} c(x) \mathrm{dS} .
$$

Equation (E) is oscillatory, if the ordinary differential equation

$$
\left(r^{n-1} \Phi\left(y^{\prime}\right)\right)^{\prime}+r^{n-1} \tilde{c}(r) \Phi(y)=0
$$

is oscillatory.

Oscillation criteria for equation (1.1) can be found in [3, 4, 5, 9, 11, 12, 13].

The disadvantage of the criteria in Theorems A and B lies in the fact that preferring integration over the balls in $\mathbb{R}^{n}$ we loose the information about the distribution of the potential $c(x)$ over the sphere $S(r)$. However, the distribution of potential over the sphere may be substantial in the cases when Theorem B is not applicable. If the function $\tilde{c}(x)$ is sufficiently small, equation (1.1) is nonoscillatory, but equation (E) still may be oscillatory.

Philos [21] used a class of functions $H(t, s)$ to obtain oscillation criteria for linear second order Sturm-Liouville differential equation. This technique, usually referred as averaging technique, has been elaborated and extended e.g. in [10, 13, 24] also for other types of ordinary differential equations. Let us point out the paper [24], where the usual condition $\frac{\partial H(t, s)}{\partial s} \leq 0$ is relaxed.

The aim of this paper is to extend the averaging technique also for the partial differential equation (E) and obtain new oscillation criteria, which can remove the disadvantage of Theorems A and B. It is also showed, that this technique allows obtain oscillation criteria not only for the exterior of a ball, but also for different types of unbounded domains.

The paper is divided into three sections. Main results and comments are presented in the next section. The last section contains proofs of theorems. 


\section{Main Results}

First let us present a direct extension of [24, Theorem 1] to the case of equation (E).

Theorem 2.1. Let $H(t, x) \in C(D,[0, \infty))$, and $\rho(x) \in C^{1}\left(\Omega\left(t_{0}\right),(0, \infty)\right)$ be such that the function $H(t, x)$ has a continuous partial derivative with respect to $x_{i}$ $(i=1 . . n)$ on $D_{0}$ and the following conditions hold

(i) $H(t, x)=0$ iff $t=\|x\|$

(ii) There exists function $k(s) \in C\left(\left[t_{0}, \infty\right),(0, \infty)\right)$ such that the function

$f(t, s)=k(s) \int_{S(s)} H(t, x) \mathrm{dS}$ is nonincreasing with respect to $s$ for every $t \geq s \geq t_{0}$.

(iii) The vector-valued function $\boldsymbol{h}(t, x)$ defined on $D_{0}$ by

$$
\boldsymbol{h}(t, x)=\nabla H(t, x)+\frac{H(t, x)}{\rho(x)} \nabla \rho(x)
$$

satisfies

$$
\int_{\Omega\left(t_{0}, t\right)} H^{1-p}(t, x)\|\boldsymbol{h}(t, x)\|^{p} \rho(x) \mathrm{d} x<\infty
$$

for $t>t_{0}$.

If

$$
\begin{aligned}
\limsup _{t \rightarrow \infty} & \left(\int_{S\left(t_{0}\right)} H(t, x) \mathrm{d} S\right)^{-1} \\
& \times \int_{\Omega\left(t_{0}, t\right)}\left[H(t, x) \rho(x) c(x)-\frac{\|\boldsymbol{h}(t, x)\|^{p} \rho(x)}{p^{p} H^{p-1}(t, x)}\right] \mathrm{d} x=\infty,
\end{aligned}
$$

then (E) is oscillatory.

The following theorem is a variant of the preceding one. In contrast to Theorem 2.1 the function $H(t, x)$ is not necessary to be positive for $t_{0} \leq\|x\|<t$ in theorems bellow, but can attain also zero values. This allows to eliminate "bad parts" of the potential $c(x)$ from our considerations. We will use the following additional notation

$$
\begin{aligned}
\Omega_{0, t}(a, b) & =\left\{x \in \mathbb{R}^{n}: a \leq\|x\| \leq b, H(t, x) \neq 0\right\}, \\
S_{0, t}(a) & =\left\{x \in \mathbb{R}^{n}:\|x\|=a, H(t, x) \neq 0\right\} .
\end{aligned}
$$

This allows us to exclude the parts of the sets $\Omega(a, b)$ and $S(a)$, where the function $H(t, x)$ equals zero, from the area of integration.

Theorem 2.2. Let $H(t, x) \in C(D,[0, \infty))$, and $\rho(x) \in C^{1}\left(\Omega\left(t_{0}\right),(0, \infty)\right)$ be such that that the function $H(t, x)$ has a continuous partial derivative with respect to $x_{i}$ $(i=1 . . n)$ on $D_{0}$ and the following conditions hold

(i) If $\|x\|=t \geq t_{0}$, then $H(t, x)=0$

(ii) If $H(t, x)=0$ for some $(t, x) \in D_{0}$, then $\|\nabla H(t, x)\|=0$ 
(iii) There exists function $k(s) \in C\left(\left[t_{0}, \infty\right),(0, \infty)\right)$ such that the function $f(t, s):=k(s) \int_{S(s)} H(t, x) \mathrm{dS}=k(s) \int_{S_{0, t}(s)} H(t, x) \mathrm{dS}$ is positive and nonincreasing with respect to $s$ for every $t>s \geq t_{0}$.

(iv) The vector-valued function $\boldsymbol{h}(t, x)$ defined on $D_{0}$ by (2.1) satisfies

$$
\int_{\Omega_{0, t}\left(t_{0}, t\right)} H^{1-p}(t, x)\|\boldsymbol{h}(t, x)\|^{p} \rho(x) \mathrm{d} x<\infty
$$

for $t>t_{0}$.

If

$$
\begin{aligned}
\limsup _{t \rightarrow \infty} & \left(\int_{S\left(t_{0}\right)} H(t, x) \mathrm{d} S\right)^{-1} \\
& \times \int_{\Omega_{0, t}\left(t_{0}, t\right)}\left[H(t, x) \rho(x) c(x)-\frac{\|\boldsymbol{h}(t, x)\|^{p} \rho(x)}{p^{p} H^{p-1}(t, x)}\right] \mathrm{d} x=\infty
\end{aligned}
$$

then (E) is oscillatory.

Remark 2.1. Condition (iii) claims that the set $S_{0, t}(s)$ is nonempty for every $t$ satisfying $t_{0}<s<t$. Hence The function $H(t, x)$ has parts with positive values on every sphere centered in the origin.

Remark 2.2. Under (2.2) we understand that the function $g(t, s)$ defined for $t_{0}<s<t$ by

$$
g(t, s):=\int_{S_{0, t}(s)} H^{1-p}(t, x) \rho(x)\|\boldsymbol{h}(t, x)\|^{p} \mathrm{dS}
$$

is integrable with respect to $s$ over the interval $\left(t_{0}, t\right)$. (The point $t$ may be a singular point of the integral, since $H(t, x)=0$ for $\|x\|=t$.) A similar commentary explains also, how to understand (2.4).

Remark 2.3. Let $\Omega \subset \Omega\left(t_{0}\right)$ be unbounded domain with smooth boundary $\partial \Omega$. If in addition to the conditions of Theorem 2.2 the function $H(t, x)$ vanishes outside $\Omega$ and both $H(t, x)$ and $\|\nabla H(t, x)\|$ vanishes on $\partial \Omega$ for every $t \geq t_{0}$, then it follows that equation (E) is oscillatory in $\Omega$. Hence Theorem 2.2 can be used to formulate explicit oscillation criteria on different types of domains, than exterior of the ball. This situation cannot be covered by Theorem B. Remark also that Kneser-type criteria for oscillation and nonoscillation of linear PDE in various types of unbounded domain can be found in [1]. Examples of the oscillation criteria on the half-plane are given bellow.

The following Corollary is an immediate consequence of Theorem 2.2.

Corollary 2.1. Let the assumptions (i) - (iv) of Theorem 2.2 holds. If

$$
\limsup _{t \rightarrow \infty}\left(\int_{S\left(t_{0}\right)} H(t, x) \mathrm{d} S\right)^{-1} \int_{\Omega_{0, t}\left(t_{0}, t\right)} \frac{\|\boldsymbol{h}(t, x)\|^{p} \rho(x)}{H^{p-1}(t, x)} \mathrm{d} x<\infty
$$


and

$$
\limsup _{t \rightarrow \infty}\left(\int_{S\left(t_{0}\right)} H(t, x) \mathrm{d} S\right)^{-1} \int_{\Omega\left(t_{0}, t\right)} H(t, x) \rho(x) c(x) \mathrm{d} x=\infty,
$$

then (E) is oscillatory.

The following theorem extends [24, Theorem 2].

Theorem 2.3. Let the functions $H, h, k$ and $\rho$ satisfy the hypotheses (i)-(iv) of Theorem 2.2. Suppose also that

$$
0<\inf _{s \geq t_{0}}\left\{\liminf _{t \rightarrow \infty} \frac{k(s) \int_{S(s)} H(t, x) \mathrm{dS}}{k\left(t_{0}\right) \int_{S\left(t_{0}\right)} H(t, x) \mathrm{d} S}\right\}
$$

and (2.7) holds. If there exists a function $A \in C\left(\Omega\left(t_{0}\right), \mathbb{R}\right)$ such that

$$
\begin{gathered}
\inf _{t \in(T, \infty)}\left\{\left(\int_{S(T)} H(t, x) \mathrm{d} S\right)^{-1} \int_{\Omega_{0, t}(T, t)}[H(t, x) \rho(x) c(x)\right. \\
\left.\left.-\frac{\|\boldsymbol{h}(t, x)\|^{p} \rho(x)}{p^{p} H^{p-1}(t, x)}\right] \mathrm{d} x\right\} \geq A(T)
\end{gathered}
$$

for $T \geq t_{0}$ and

$$
\int_{t_{0}}^{\infty}\left(A_{+}(T)\right)^{q} \widehat{\rho}^{1-q}(T) k^{-1}(T) \mathrm{d} T=\infty,
$$

where $A_{+}(T)=\max \{A(T), 0\}$ and

$$
\widehat{\rho}(T)=\sup _{t>T}\left\{\left(\int_{S(T)} H(t, x) \mathrm{d} S\right)^{-1} \int_{S(T)} \rho(x) H(t, x) \mathrm{d} S\right\},
$$

then (E) is oscillatory.

Remark 2.4. The supremum in (2.12) always exists, since

$$
\left(\int_{S(T)} H(t, x) \mathrm{d} S\right)^{-1} \int_{S(T)} \rho(x) H(t, x) \mathrm{d} S \leq \max _{x \in S(T)}\{\rho(x)\} .
$$

Remark 2.5. Comparing Theorem 2.3 with Theorem 2 of [24] we see that in the case of ordinary differential equations is the condition (2.10) replaced by a weaker condition where $\lim \sup _{t \rightarrow \infty}$ stays instead of $\inf _{t \in(T, \infty)}$. The reason, why we need the stronger condition (2.10) instead is the following. In the proof of Theorem 2.3 we estimate the function $A(T)$ from above with help of solution of Riccati equation - see (3.19) below. This bound does not depend on the value of $t$ in the case of ODE, however depends on $t$ in the case of equation (E).

The following theorem extends [24, Theorem 3] 
Theorem 2.4. Let the functions $H, h, k$ and $\rho$ satisfy the hypotheses (i)-(iv) of Theorem 2.2. Suppose also that (2.9) and

$$
\liminf _{t \rightarrow \infty}\left(\int_{S\left(t_{0}\right)} H(t, x) \mathrm{d} S\right)^{-1} \int_{\Omega\left(t_{0}, t\right)} H(t, x) \rho(x) c(x) \mathrm{d} x<\infty .
$$

If there exists a function $A \in C\left(\Omega\left(t_{0}\right), \mathbb{R}\right)$ such that (2.10) and (2.11)hold, then (E) is oscillatory.

Example 2.1. Consider the Schrödinger partial differential equation (L) in $\mathbb{R}^{2}$, i.e., $n=p=2$. For $\lambda>1$ define the functions $H, k$ and $\rho$ as follows:

$$
\begin{aligned}
\rho(x) & \equiv 1 \quad \text { for } x \in \mathbb{R}^{2} \\
k(s) & =\frac{1}{s} \quad \text { for } s>1 \\
H(t, x) & = \begin{cases}(t-r)^{\lambda} \sin ^{2} \phi & \phi \in[0, \pi) \\
0 & \phi \in[\pi, 2 \pi),\end{cases}
\end{aligned}
$$

where $r$ and $\phi$ are the radial and the polar coordinates of the point $x \in \mathbb{R}^{2}$. It is easy to see that $S_{t, 0}(s)$ is the top half-circle with radius $s<t$ and $\int_{S(s)} H(t, x) \mathrm{dS}=$ $\frac{\pi}{2}(t-s)^{\lambda} s=O\left(t^{\lambda}\right)$. Since $\rho(x) \equiv 1, \boldsymbol{h}(t, x)=\nabla H(t, x)$ holds and consequently

$$
\|\boldsymbol{h}(t, x)\|^{2}= \begin{cases}\lambda^{2}(t-r)^{2 \lambda-2} \sin ^{4} \phi+4 \frac{(t-r)^{2 \lambda}}{r^{2}} \sin ^{2} \phi \cos ^{2} \phi & \phi \in[0, \pi) \\ 0 & \phi \in[\pi, 2 \pi)\end{cases}
$$

Direct computation shows

$$
H^{-1}(t, x)\|\boldsymbol{h}(t, x)\|^{2}=\lambda^{2}(t-r)^{\lambda-2} \sin ^{2} \phi+4 \frac{(t-r)^{\lambda}}{r^{2}} \cos ^{2} \phi
$$

for $x \in \Omega_{0, t}\left(t_{0}\right)$ and (2.4) clearly holds. Further (2.5) has the form

$$
\begin{aligned}
\limsup _{t \rightarrow \infty} t^{-\lambda} & \int_{M(t)}\left[c(x(r, \phi))(t-r)^{\lambda} \sin ^{2} \phi\right. \\
& \left.-\frac{\lambda^{2}}{4}(t-r)^{\lambda-2} \sin ^{2} \phi-\frac{(t-r)^{\lambda}}{r^{2}} \cos ^{2} \phi\right] \mathrm{d} x=\infty,
\end{aligned}
$$

where $M(t)=\left\{\left(x_{1}, x_{2}\right) \in \mathbb{R}^{2}: 1 \leq x_{1}^{2}+x_{2}^{2} \leq t^{2}, x_{2}>0\right\}$. Since

$$
\begin{aligned}
\lim _{t \rightarrow \infty} t^{-\lambda} \int_{M(t)}(t-r)^{\lambda-2} \sin ^{2} \phi \mathrm{d} x & =\lim _{t \rightarrow \infty} t^{-\lambda} \frac{\pi}{2} \int_{1}^{t} r(t-r)^{\lambda-2} \mathrm{~d} r \\
& \leq \lim _{t \rightarrow \infty} t^{-\lambda} \frac{\pi}{2} \int_{1}^{t} t(t-r)^{\lambda-2} \mathrm{~d} r \\
& =\frac{\pi}{2} \frac{1}{\lambda-1} \lim _{t \rightarrow \infty} t^{1-\lambda}(t-1)^{\lambda-1}<\infty
\end{aligned}
$$

is $(2.14)$ equivalent to

$$
\limsup _{t \rightarrow \infty} t^{-\lambda} \int_{M(t)}\left[c(x(r, \phi))(t-r)^{\lambda} \sin ^{2} \phi-\frac{(t-r)^{\lambda}}{r^{2}} \cos ^{2} \phi\right] \mathrm{d} x(r, \phi)=\infty .
$$


Hence (2.14) is sufficient for (L) to be oscillatory on the half-plane $x_{2} \geq 0$.

Example 2.2. Lut us consider the same equation as in the Example 2.1. Let us change the function $\rho(x)$ into $\rho(x)=\frac{1}{\|x\|}=\frac{1}{r}$. The computation in polar coordinates yields

$$
\begin{aligned}
\|\boldsymbol{h}(t, x)\|^{2}= & \lambda^{2}(t-r)^{2 \lambda-2} \sin ^{4} \phi+2 \lambda(t-r)^{2 \lambda-1} r^{-1} \sin ^{4} \phi \\
& +(t-r)^{2 \lambda} r^{-2} \sin ^{4} \phi+4(t-r)^{2 \lambda} r^{-2} \sin ^{2} \phi \cos ^{2} \phi
\end{aligned}
$$

for $\phi \in[0, \pi)$ and $\|\boldsymbol{h}(t, x)\|^{2}=0$ otherwise. As in the preceeding example, (2.4) holds. Further integrating in polar coordinates we ensure that (2.7) holds. Then the condition

$$
\limsup _{t \rightarrow \infty} t^{-\lambda} \int_{M(t)} c(x(r, \phi))(t-r)^{\lambda} r^{-1} \sin ^{2} \phi \mathrm{d} x(r, \phi)=\infty
$$

is a sufficient condition for oscillation of equation (E) on the half-plane $x_{2} \geq 0$.

Remark 2.6. In contrast to the results in Theorems A and B, the conditions in Examples 2.1 and 2.2 are not affected by the behavior of the function $c(x)$ on the half-plane $x_{2} \leq 0$, which may be "relatively bad".

\section{Proofs}

Proof of Theorem 2.1. Suppose that (E) is not oscillatory. There exits $T \geq t_{0}$, such that (E) has a solution $u$ positive on $\Omega(T)$. The Riccati-type vector variable

$$
\boldsymbol{w}(x):=\rho(x) \frac{\left.\|\nabla u(x)\|\right|^{p-2} \nabla u(x)}{\Phi(u(x))}
$$

is well-defined on $\Omega(T)$ and satisfies

$$
\operatorname{div} \boldsymbol{w}(x)=\rho(x) \frac{\Delta_{p} u}{\Phi(u)}+\frac{\|\nabla u\|^{p-2}}{\Phi(u)}\langle\nabla u, \nabla \rho(x)\rangle-(p-1) \rho(x) \frac{\|\nabla u\|^{p}}{|u|^{p}} .
$$

The application of (E) and (3.1) gives

$$
\operatorname{div} \boldsymbol{w}(x)=-\rho(x) c(x)+\frac{1}{\rho(x)}\langle\boldsymbol{w}(x), \nabla \rho(x)\rangle-(p-1) \rho^{1-q}(x)\|\boldsymbol{w}(x)\|^{q}
$$

and equivalently

$$
\rho(x) c(x)=-\operatorname{div} \boldsymbol{w}(x)+\frac{1}{\rho(x)}\langle\boldsymbol{w}(x), \underset{8}{\nabla} \rho(x)\rangle-(p-1) \rho^{1-q}(x)\|\boldsymbol{w}(x)\|^{q}
$$


for $x \in \Omega(T)$. Multiplication of this equality by the factor $H(t, x)$ and integration over $\Omega(T, t)$ for $t>T$ yields

$$
\begin{aligned}
\int_{\Omega(T, t)} & H(t, x) \rho(x) c(x) \mathrm{d} x=-\int_{\Omega(T, t)} H(t, x) \operatorname{div} \boldsymbol{w}(x) \mathrm{d} x \\
& +\int_{\Omega(T, t)} H(t, x) \frac{1}{\rho(x)}\langle\boldsymbol{w}(x), \nabla \rho(x)\rangle \mathrm{d} x \\
& -\int_{\Omega(T, t)} H(t, x)(p-1) \rho^{1-q}(x)\|\boldsymbol{w}(x)\|^{q} \mathrm{~d} x .
\end{aligned}
$$

From here we conclude that

$$
\begin{aligned}
\int_{\Omega(T, t)} & H(t, x) \rho(x) c(x) \mathrm{d} x=-\int_{\Omega(T, t)} \operatorname{div}(H(t, x) \boldsymbol{w}(x)) \mathrm{d} x \\
& +\int_{\Omega(T, t)}\langle\nabla H(t, x), \boldsymbol{w}(x)\rangle \mathrm{d} x+\int_{\Omega(T, t)} H(t, x) \frac{1}{\rho(x)}\langle\boldsymbol{w}(w), \nabla \rho(x)\rangle \mathrm{d} x \\
& -\int_{\Omega(T, t)} H(t, x)(p-1) \rho^{1-q}(x)\|\boldsymbol{w}(x)\|^{q} \mathrm{~d} x .
\end{aligned}
$$

Application of Gauss-Ostrogradski theorem, the property (i) of the function $H(t, x)$ and (2.1) gives

$$
\begin{aligned}
\int_{\Omega(T, t)} & H(t, x) \rho(x) c(x) \mathrm{d} x=\int_{S(T)} H(t, x)\langle\boldsymbol{w}(x), \nu\rangle \mathrm{d} x \\
& +\int_{\Omega(T, t)}\langle\boldsymbol{h}(t, x), \boldsymbol{w}(x)\rangle \mathrm{d} x-\int_{\Omega(T, t)} H(t, x)(p-1) \rho^{1-q}(x)\|\boldsymbol{w}(x)\|^{q} \mathrm{~d} x
\end{aligned}
$$

where $\nu$ is the normal unit vector. From here and from the Young inequality

$$
(p-1)\|\boldsymbol{X}\|^{q}-p\langle\boldsymbol{X}, \boldsymbol{Y}\rangle+\|\boldsymbol{Y}\|^{p} \geq 0
$$

for $\boldsymbol{X}=\boldsymbol{w}(x) H^{\frac{1}{q}}(t, x) \rho^{-\frac{1}{p}}(x)$ and $\boldsymbol{Y}=\boldsymbol{h}(t, x) \rho^{\frac{1}{p}}(x) p^{-1} H^{\frac{1-p}{p}}(t, x)$ it follows

$$
\begin{aligned}
\int_{\Omega(T, t)} & H(t, x) \rho(x) c(x) \mathrm{d} x \\
& \leq \int_{S(T)} H(t, x)\langle\boldsymbol{w}(x), \nu\rangle \mathrm{d} x+\int_{\Omega(T, t)} \frac{\|\boldsymbol{h}(t, x)\|^{p} \rho(x)}{p^{p} H^{p-1}(t, x)} \mathrm{d} x .
\end{aligned}
$$

which is equivalent to

$$
\int_{\Omega(T, t)}\left[H(t, x) \rho(x) c(x)-\frac{\|\boldsymbol{h}(t, x)\|^{p} \rho(x)}{p^{p} H^{p-1}(t, x)}\right] \mathrm{d} x \leq \int_{S(T)} H(t, x)\langle\boldsymbol{w}(x), \nu\rangle \mathrm{d} x
$$

Hence

$$
\int_{\Omega(T, t)}\left[H(t, x) \rho(x) c(x)-\frac{\|\boldsymbol{h}(t, x)\|^{p} \rho(x)}{p^{p} H^{p-1}(t, x)}\right] \mathrm{d} x \leq w^{*}(T) \int_{S(T)} H(t, x) \mathrm{d} S,
$$


where $w^{*}(T)=\max _{x \in S(T)}\{\|\boldsymbol{w}(x)\|\}$. Using (3.4) we are able to estimate the integral from the condition $(2.3)$

$$
\begin{aligned}
\int_{\Omega\left(t_{0}, t\right)} & {\left[H(t, x) \rho(x) c(x)-\frac{\|\boldsymbol{h}(t, x)\|^{p} \rho(x)}{p^{p} H^{p-1}(t, x)}\right] \mathrm{d} x } \\
\leq & \int_{\Omega\left(t_{0}, T\right)} H(t, x) \rho(x) c(x) \mathrm{d} x+w^{*}(T) \int_{S(T)} H(t, x) \mathrm{d} \mathrm{S} \\
\leq & \int_{t_{0}}^{T}\left[\int_{S(s)} H(t, x) \mathrm{d} \mathrm{S}\right] k(s) \frac{\rho^{*}(s) c^{*}(s)}{k(s)} \mathrm{d} s+\frac{w^{*}(T)}{k(T)} k(T) \int_{S(T)} H(t, x) \mathrm{d} \mathrm{S}
\end{aligned}
$$

for $t>T$ where $\rho^{*}(s)=\max _{x \in S(s)}\{\rho(x)\}$ and $c^{*}(s)=\max _{x \in S(s)}\{|c(x)|\}$. Since $f(t, s):=k(s) \int_{S(s)} H(t, x) \mathrm{dS}$ is a nonincreasing function with respect to $s$, the above inequality implies

$$
\begin{aligned}
& \int_{\Omega\left(t_{0}, t\right)} {\left[H(t, x) \rho(x) c(x)-\frac{\|\boldsymbol{h}(t, x)\|^{p} \rho(x)}{p^{p} H^{p-1}(t, x)}\right] \mathrm{d} x } \\
& \leq k\left(t_{0}\right)\left[\int_{S\left(t_{0}\right)} H(t, x) \mathrm{d} \mathrm{S}\right]\left[\int_{t_{0}}^{T} \frac{\rho^{*}(s) c^{*}(s)}{k(s)} \mathrm{d} s+\frac{w^{*}(T)}{k(T)}\right]
\end{aligned}
$$

and hence

$$
\begin{aligned}
\left(\int_{S\left(t_{0}\right)} H(t, x) \mathrm{d} S\right)^{-1} & \int_{\Omega\left(t_{0}, t\right)}\left[H(t, x) \rho(x) c(x)-\frac{\|\boldsymbol{h}(t, x)\|^{p} \rho(x)}{p^{p} H^{p-1}(t, x)}\right] \mathrm{d} x \\
\leq & k\left(t_{0}\right) \int_{t_{0}}^{T} \frac{\rho^{*}(s) c^{*}(s)}{k(s)} \mathrm{d} s+\frac{k\left(t_{0}\right) w^{*}(T)}{k(T)}
\end{aligned}
$$

for large $t$, which contradicts (2.3).

Proof of Theorem 2.2. Assume the contradiction. As in the proof of Theorem 2.1 we conclude (3.2) for $t>T$, where $w$ is the solution of Riccati-type equation, defined on $\Omega(T)$. Since $H(t, x)=\|\boldsymbol{h}(t, x)\|=0$ for $x \in \Omega(T, t) \backslash \Omega_{0, t}(T, t)$, we have

$$
\begin{aligned}
\int_{\Omega(T, t)}\langle\boldsymbol{h}(t, x), \boldsymbol{w}(x)\rangle \mathrm{d} x- & \int_{\Omega(T, t)} H(t, x)(p-1) \rho^{1-q}(x)\|\boldsymbol{w}(x)\|^{q} \mathrm{~d} x \\
= & \int_{\Omega_{0, t}(T, t)}[\langle\boldsymbol{h}(t, x), \boldsymbol{w}(x)\rangle \\
& \left.\quad-H(t, x)(p-1) \rho^{1-q}(x)\|\boldsymbol{w}(x)\|^{q}\right] \mathrm{d} x .
\end{aligned}
$$


The following relation we obtain from (3.5) and from Hölder inequality

$$
\begin{aligned}
\int_{\Omega(T, t)}\langle\boldsymbol{h}( & t, x), \boldsymbol{w}(x)\rangle \mathrm{d} x-\int_{\Omega(T, t)} H(t, x)(p-1) \rho^{1-q}(x)\|\boldsymbol{w}(x)\|^{q} \mathrm{~d} x \\
= & \int_{T}^{t}\left[\int_{S_{0, t}(s)}\langle\boldsymbol{h}(t, x), \boldsymbol{w}(x)\rangle \mathrm{d} \mathrm{S}\right. \\
& \left.-\int_{S_{0, t}(s)} H(t, x)(p-1) \rho^{1-q}(x)\|\boldsymbol{w}(x)\|^{q} \mathrm{~d} \mathrm{~S}\right] \mathrm{d} s \\
\leq & \int_{T}^{t}\left[\left(\int_{S_{0, t}(s)} H^{1-p}(t, x) \rho(x)\|\boldsymbol{h}(t, x)\|^{p} \mathrm{dS}\right)^{\frac{1}{p}}\right. \\
& \times\left(\int_{S_{0, t}(s)} H(t, x) \rho^{1-q}(x)\|\boldsymbol{w}(x)\|^{q} \mathrm{dS}\right)^{\frac{1}{q}} \\
& \left.-\int_{S_{0, t}(s)} H(t, x)(p-1) \rho^{1-q}(x)\|\boldsymbol{w}(x)\|^{q} \mathrm{dS}\right] \mathrm{d} s
\end{aligned}
$$

Application of Young inequality (3.3) gives

$$
\begin{aligned}
\int_{\Omega(T, t)}\langle\boldsymbol{h}(t, x), \boldsymbol{w}(x)\rangle \mathrm{d} x & -\int_{\Omega(T, t)} H(t, x)(p-1) \rho^{1-q}(x)\|\boldsymbol{w}(x)\|^{q} \mathrm{~d} x \\
& \leq \int_{T}^{t} p^{-p} \int_{S_{0, t}(s)} H^{1-p}(t, x) \rho(x)\|\boldsymbol{h}(t, x)\|^{p} \mathrm{~d} \mathrm{~d} \mathrm{~d} s \\
& =\int_{\Omega_{0, t}(T, t)} p^{-p} H^{1-p}(t, x) \rho(x)\|\boldsymbol{h}(t, x)\|^{p} \mathrm{~d} x
\end{aligned}
$$

Combining this inequality with (3.2) we conclude

$$
\begin{aligned}
& \int_{\Omega_{0, t}(T, t)} {\left[H(t, x) \rho(x) c(x)-p^{-p} \rho(x) H^{1-p}(t, x)\|\boldsymbol{h}(t, x)\|^{p}\right] \mathrm{d} x } \\
& \leq \int_{S(T)} H(t, x)\langle\boldsymbol{w}(x), \nu\rangle \mathrm{d} x
\end{aligned}
$$

and similarly as in the proof of Theorem 2.1 we obtain

$$
\begin{aligned}
\int_{\Omega_{0, t}\left(t_{0}, t\right)} & {\left[H(t, x) \rho(x) c(x)-p^{-p} \rho(x) H^{1-p}(t, x)\|\boldsymbol{h}(t, x)\|^{p}\right] \mathrm{d} x } \\
& \leq \int_{\Omega\left(t_{0}, T\right)} H(t, x) \rho(x) c(x) \mathrm{d} x+\int_{S(T)} H(t, x)\langle\boldsymbol{w}(x), \boldsymbol{\nu}\rangle \mathrm{d} S \\
& \leq \int_{t_{0}}^{T}\left[\int_{S(s)} H(t, x) \mathrm{dS}\right] k(s) \frac{\rho^{*}(s) c^{*}(s)}{k(s)} \mathrm{d} s+w^{*}(T) \int_{S(T)} H(t, x) \mathrm{d} S \\
& \leq k\left(t_{0}\right)\left[\int_{S\left(t_{0}\right)} H(t, x) \mathrm{dS}\right]\left[\int_{t_{0}}^{T} \frac{\rho^{*}(s) c^{*}(s)}{k(s)} \mathrm{d} s+\frac{w^{*}(T)}{k(T)}\right],
\end{aligned}
$$


where $w^{*}(s), \rho^{*}(s)$ and $c^{*}(s)$ are the same as in the proof of Theorem 2.1. The last inequality contradicts (2.5). The proof is complete.

Lemma 3.1. Let the functions $H, h, k$ and $\rho$ satisfy the hypothesis (i)-(iv) of Theorem 2.2. Suppose that (2.7), (2.9) and (2.10) holds. Let $u$ be solution of (E) which is positive on $\Omega\left(T_{0}\right)$ for some $T_{0} \geq t_{0}$ and $\boldsymbol{w}(x)$ be the corresponding Riccati variable defined on $\Omega\left(T_{0}\right)$ by (3.1). Then

$$
\liminf _{t \rightarrow \infty} \int_{T_{0}}^{t} \frac{\int_{S(s)} H(t, x) \rho^{1-q}(x)\|\boldsymbol{w}(x)\|^{q} \mathrm{dS}}{k(s) \int_{S(s)} H(t, x) \mathrm{dS}} \mathrm{d} s<\infty .
$$

Proof. Let us denote

$$
\begin{aligned}
F(t) & =\left(\int_{S\left(T_{0}\right)} H(t, x) \mathrm{d} S\right)^{-1} \int_{\Omega\left(T_{0}, t\right)}\|\boldsymbol{h}(t, x)\| \cdot\|\boldsymbol{w}(x)\| \mathrm{d} x \\
G(t) & =\left(\int_{S\left(T_{0}\right)} H(t, x) \mathrm{d} S\right)^{-1}(p-1) \int_{\Omega\left(T_{0}, t\right)} H(t, x) \rho^{1-q}(x)\|\boldsymbol{w}(x)\|^{q} \mathrm{~d} x
\end{aligned}
$$

for $t>T_{0}$. As in the proof of Theorem 2.2 we conclude (3.2) and hence

$$
\begin{aligned}
G(t)-F(t) \leq & \left(\int_{S\left(T_{0}\right)} H(t, x) \mathrm{dS}\right)^{-1} \\
& \times\left[\int_{S\left(T_{0}\right)} H(t, x)\|\boldsymbol{w}(x)\| \mathrm{dS}-\int_{\Omega\left(T_{0}, t\right)} H(t, x) \rho(x) c(x) \mathrm{d} x\right] \\
\leq & w^{*}\left(T_{0}\right)-\left(\int_{S\left(T_{0}\right)} H(t, x) \mathrm{dS}\right)^{-1} \int_{\Omega\left(T_{0}, t\right)} H(t, x) \rho(x) c(x) \mathrm{d} x
\end{aligned}
$$

holds for every $t>T_{0}$, where $w^{*}(t)$ has been defined in the proof of Theorem 2.1. Hence by (2.10)

$$
\liminf _{t \rightarrow \infty}[G(t)-F(t)] \leq w^{*}\left(T_{0}\right)-A\left(T_{0}\right)<\infty .
$$

Suppose that (3.7) does not hold. Then

$$
\lim _{t \rightarrow \infty} \int_{T_{0}}^{t} \frac{\int_{S(s)} H(t, x) \rho^{1-q}(x)\|\boldsymbol{w}(x)\|^{q} \mathrm{dS}}{k(s) \int_{S(s)} H(t, x) \mathrm{d} S} \mathrm{~d} s=\infty .
$$

According to (2.9) there exists $\eta \in \mathbb{R}$ such that

$$
0<\eta<\inf _{s \geq t_{0}}\left\{\liminf _{t \rightarrow \infty} \frac{k(s) \int_{S(s)} H(t, x) \mathrm{dS}}{k\left(t_{0}\right) \int_{S\left(t_{0}\right)} H(t, x) \mathrm{dS}}\right\}
$$

and for every $\mu \in \mathbb{R}^{+}$there exists $T_{1}>T_{0}$ such that

$$
\int_{T_{0}}^{t} \frac{(p-1) \int_{S(s)} H(t, x) \rho^{1-q}(x)\|\boldsymbol{w}(x)\|^{q} \mathrm{~d} S}{k(s) \int_{S(s)} H(t, x) \mathrm{d} S} \mathrm{~d} s \geq \frac{\mu}{\eta k\left(T_{0}\right)}
$$


for every $t \geq T_{1}$. Further there exists $T_{2}>T_{1}$ such that

$$
\frac{k\left(T_{1}\right) \int_{S\left(T_{1}\right)} H(t, x) \mathrm{d} S}{k\left(t_{0}\right) \int_{S\left(t_{0}\right)} H(t, x) \mathrm{d} S}>\eta
$$

for all $t \geq T_{2}$. From the definition of the function $G(t)$ it follows that for $t \geq T_{2}$

$$
\begin{aligned}
G(t)= & \left(\int_{S\left(T_{0}\right)} H(t, x) \mathrm{dS}\right)^{-1} \int_{T_{0}}^{t}\left[\left(k(s) \int_{S(s)} H(t, x) \mathrm{dS}\right)\right. \\
& \left.\times \frac{(p-1) \int_{S(s)} H(t, x) \rho^{1-q}(x)\|\boldsymbol{w}(x)\|^{q} \mathrm{~d} S}{k(s) \int_{S(s)} H(t, x) \mathrm{dS}}\right] \mathrm{d} s
\end{aligned}
$$

holds. Integration by parts and the property (i) of the function $H(t, x)$ imply

$$
\begin{aligned}
G(t) \geq & \left(\int_{S\left(T_{0}\right)} H(t, x) \mathrm{dS}\right)^{-1} \int_{T_{0}}^{t}\left[-\frac{\partial}{\partial s}\left(k(s) \int_{S(s)} H(t, x) \mathrm{d} S\right)\right. \\
& \left.\times\left(\int_{T_{0}}^{s} \frac{(p-1) \int_{S(\xi)} H(t, x) \rho^{1-q}(x)\|\boldsymbol{w}(x)\|^{q} \mathrm{dS}}{k(\xi) \int_{S(\xi)} H(t, x) \mathrm{d} S} \mathrm{~d} \xi\right)\right] \mathrm{d} s
\end{aligned}
$$

and in view of (iii)

$$
\begin{aligned}
G(t) \geq & \left(\int_{S\left(T_{0}\right)} H(t, x) \mathrm{d} S\right)^{-1} \int_{T_{1}}^{t}\left[-\frac{\partial}{\partial s}\left(k(s) \int_{S(s)} H(t, x) \mathrm{d} S\right)\right. \\
& \left.\times\left(\int_{T_{0}}^{s} \frac{(p-1) \int_{S(\xi)} H(t, x) \rho^{1-q}(x)\|\boldsymbol{w}(x)\|^{q} \mathrm{~d} S}{k(\xi) \int_{S(\xi)} H(t, x) \mathrm{dS}} \mathrm{d} \xi\right)\right] \mathrm{d} s .
\end{aligned}
$$

Application of (3.12) gives

$$
\begin{aligned}
G(t) & \geq\left(\int_{S\left(T_{0}\right)} H(t, x) \mathrm{dS}\right)^{-1} \frac{\mu}{\eta k\left(T_{0}\right)} \int_{T_{1}}^{t}-\frac{\partial}{\partial s}\left(k(s) \int_{S(s)} H(t, x) \mathrm{d} S\right) \mathrm{d} s \\
& \geq \frac{\mu k\left(T_{1}\right) \int_{S\left(T_{1}\right)} H(t, x) \mathrm{d} S}{\eta k\left(T_{0}\right) \int_{S\left(T_{0}\right)} H(t, x) \mathrm{dS}} .
\end{aligned}
$$

In view of (iii)

$$
G(t) \geq \frac{\mu k\left(T_{1}\right) \int_{S\left(T_{1}\right)} H(t, x) \mathrm{d} S}{\eta k\left(t_{0}\right) \int_{S\left(t_{0}\right)} H(t, x) \mathrm{d} S}
$$

and (3.13) implies

$$
G(t) \geq \mu
$$

for every $t \geq T_{2}$. Since $\mu$ has been chosen arbitrary, $\lim _{t \rightarrow \infty} G(t)=\infty$. Let us consider the sequence $\left\{t_{n}\right\}_{n=1}^{\infty}$ of the points from $\left(T_{2}, \infty\right)$ such that $\lim _{n \rightarrow \infty} t_{n}=\infty$ and $\lim _{t \rightarrow \infty}\left[G\left(t_{n}\right)-F\left(t_{n}\right)\right]=\liminf _{t \rightarrow \infty}[G(t)-F(t)]$. In view of (3.9) there exists real constant $M$ with property

$$
G\left(t_{n}\right)-F\left(t_{n}\right) \leq M
$$


for all $n$. Hence

$$
\lim _{n \rightarrow \infty} F\left(t_{n}\right)=\lim _{n \rightarrow \infty} G\left(t_{n}\right)=\infty
$$

From (3.15) and (3.16) we obtain

$$
\frac{F\left(t_{n}\right)}{G\left(t_{n}\right)}-1 \geq-\frac{M}{G\left(t_{n}\right)}>-\frac{1}{2}
$$

for large $n$. Hence

$$
\frac{F\left(t_{n}\right)}{G\left(t_{n}\right)}>\frac{1}{2}
$$

for large $n$ and combination of this inequality with (3.16) yields

$$
\lim _{n \rightarrow \infty} \frac{F^{p}\left(t_{n}\right)}{G^{p-1}\left(t_{n}\right)}=\infty .
$$

However the definition of the function $F(t)$ and the Hölder inequality give

$$
\begin{aligned}
F(t) \leq & {\left[\left(\int_{S\left(T_{0}\right)} H(t, x) \mathrm{d} S\right)^{-1} \int_{\Omega\left(T_{0}, t\right)}(p-1) H(t, x) \rho^{1-q}(x)\|\boldsymbol{w}(x)\|^{q} \mathrm{~d} x\right]^{\frac{1}{q}} } \\
& \times\left[\left(\int_{S\left(T_{0}\right)} H(t, x) \mathrm{d} S\right)^{-1}\right. \\
& \left.\times \int_{\Omega\left(T_{0}, t\right)}(p-1)^{1-p} H^{1-p}(t, x) \rho(x)\|\boldsymbol{h}(t, x)\|^{p} \mathrm{~d} x\right]^{\frac{1}{p}} \\
\leq & {[G(t)]^{\frac{1}{q}}\left[\left(\int_{S\left(T_{0}\right)} H(t, x) \mathrm{d} S\right)^{-1}\right.} \\
& \left.\times \int_{\Omega\left(T_{0}, t\right)}(p-1)^{1-p} H^{1-p}(t, x) \rho(x)\|\boldsymbol{h}(t, x)\|^{p} \mathrm{~d} x\right]^{\frac{1}{p}}
\end{aligned}
$$

and therefore

$$
\begin{aligned}
\frac{F^{p}(t)}{G^{p-1}(t)} \leq(p & -1)^{1-p}\left(\int_{S\left(T_{0}\right)} H(t, x) \mathrm{d} S\right)^{-1} \\
& \times \int_{\Omega\left(T_{0}, t\right)}(p-1)^{1-p} H^{1-p}(t, x) \rho(x)\|\boldsymbol{h}(t, x)\|^{p} \mathrm{~d} x .
\end{aligned}
$$

Since by (3.11)

$$
\frac{k\left(T_{0}\right) \int_{S\left(T_{0}\right)} H(t, x) \mathrm{d} S}{k\left(t_{0}\right) \int_{S\left(t_{0}\right)} H(t, x) \mathrm{d} S} \geq \eta
$$

for large $t$, we have

$$
\begin{aligned}
\frac{F^{p}(t)}{G^{p-1}(t)} \leq & (p-1)^{1-p} \eta^{-1}\left(k\left(t_{0}\right) \int_{S\left(t_{0}\right)} H(t, x) \mathrm{d} S\right)^{-1} \\
& \times k\left(T_{0}\right) \int_{\Omega\left(t_{0}, t\right)}(p-1)^{1-p} H^{1-p}(t, x) \rho(x)\|\boldsymbol{h}(t, x)\|^{p} \mathrm{~d} x
\end{aligned}
$$


If (3.17) would hold we obtain a contradiction with (2.7) This contradiction completes the proof.

Proof of Theorem 2.3. Suppose that equation (E) is not oscillatory and $u$ is a solution of (E) positive on $\Omega\left(T_{0}\right)$ for some $T_{0} \geq t_{0}$. Let $\boldsymbol{w}(x)$ be Riccati variable defined by (3.1). As in the proof of Theorem 2.2 we conclude (3.6) and hence by (2.10)

$$
A(T) \leq \frac{\int_{S(T)} H(t, x)\|\boldsymbol{w}(x)\| \mathrm{d} S}{\int_{S(T)} H(t, x) \mathrm{dS}}
$$

for every $t>T>T_{0}$. Hence

$$
A(T) \int_{S(T)} H(t, x) \mathrm{d} \mathrm{S} \leq \int_{S(T)} H(t, x)\|\boldsymbol{w}(x)\| \mathrm{d} \mathrm{S}
$$

for all $t>T$. Hölder inequality gives

$$
\begin{aligned}
A(T) \int_{S(T)} H(t, x) \mathrm{d} S \leq & \left(\int_{S(T)} H(t, x) \rho^{1-q}(x)\|\boldsymbol{w}(x)\|^{q} \mathrm{~d} \mathrm{~S}\right)^{\frac{1}{q}} \\
& \times\left(\int_{S(T)} H(t, x) \rho(x) \mathrm{d} S\right)^{\frac{1}{p}} .
\end{aligned}
$$

Hence

$$
\begin{array}{r}
\left(A_{+}(T)\right)^{q}\left(\int_{S(T)} H(t, x) \mathrm{d} S\right)^{q} \leq \int_{S(T)} H(t, x) \rho^{1-q}(x)\|\boldsymbol{w}(x)\|^{q} \mathrm{~d} \mathrm{~S} \\
\times\left(\int_{S(T)} H(t, x) \rho(x) \mathrm{d} S\right)^{q-1}
\end{array}
$$

and the definition of the function $\widehat{\rho}$ yields

$$
\left(A_{+}(T)\right)^{q}(\widehat{\rho}(T))^{1-q} \leq\left(\int_{S(T)} H(t, x) \mathrm{d} \mathrm{S}\right)^{-1} \int_{S(T)} H(t, x) \rho^{1-q}(x)\|\boldsymbol{w}(x)\|^{q} \mathrm{~d} \mathrm{~S}
$$

for $t>T>T_{0}$. This inequality combined with (3.7) contradicts to (2.11). The proof is complete.

Lemma 3.2. Let the functions $H, h, k$ and $\rho$ satisfy the hypothesis (i)-(iv) and of Theorem 2.2. Suppose that (2.9), (2.10) and (2.13) holds. Let $u$ and $\boldsymbol{w}$ be the same as in Lemma 3.1. Then (3.7) holds.

Proof. As in the proof of Theorem 2.2 we see that (3.2) holds. With the notation of Lemma 3.1 inequality (3.8) holds. Hence

$$
\begin{aligned}
\limsup _{t \rightarrow \infty}[G(t)-F(t)] & \leq w^{*}\left(T_{0}\right)-\liminf _{t \rightarrow \infty}\left(\int_{S\left(T_{0}\right)} H(t, x) \mathrm{dS}\right)^{-1} \\
& \times \int_{\Omega\left(t_{0}, t\right)} H(t, x) \rho(x) c(x) \mathrm{d} x \\
& \leq w^{*}\left(T_{0}\right)-A\left(T_{0}\right)<\infty .
\end{aligned}
$$


By (2.10)

$$
A\left(t_{0}\right) \leq\left(\int_{S\left(t_{0}\right)} H(t, x) \mathrm{dS}\right)^{-1} \int_{\Omega_{0, t}\left(t_{0}, t\right)}\left[H(t, x) \rho(x) c(x)-\frac{\|\boldsymbol{h}(t, x)\|^{p} \rho(x)}{p^{p} H^{p-1}(t, x)}\right] \mathrm{d} x
$$

for $t \geq t_{0}$. Hence by (2.13)

$$
\begin{aligned}
& \liminf _{t \rightarrow \infty}\left(\int_{S\left(t_{0}\right)} H(t, x) \mathrm{dS}\right)^{-1} \int_{\Omega_{0, t}\left(t_{0}, t\right)} \frac{\|\boldsymbol{h}(t, x)\|^{p} \rho(x)}{p^{p} H^{p-1}(t, x)} \mathrm{d} x \\
& \quad \leq \liminf _{t \rightarrow \infty}\left(\int_{S\left(t_{0}\right)} H(t, x) \mathrm{d} S\right)^{-1} \int_{\Omega_{0, t}\left(t_{0}, t\right)} H(t, x) \rho(x) c(x) \mathrm{d} x-A\left(t_{0}\right) \\
& \quad<\infty
\end{aligned}
$$

Let us consider the sequence $\left\{t_{n}\right\}_{n=1}^{\infty}$ in $\left(T_{0}, \infty\right)$ satisfying $\lim _{n \rightarrow \infty} t_{n}=\infty$ and

$$
\begin{aligned}
\lim _{n \rightarrow \infty} & \left(\int_{S\left(t_{0}\right)} H\left(t_{n}, x\right) \mathrm{d} S\right)^{-1} \int_{\Omega_{0, t_{n}}\left(t_{0}, t_{n}\right)} \frac{\left\|\boldsymbol{h}\left(t_{n}, x\right)\right\|^{p} \rho(x)}{p^{p} H^{p-1}\left(t_{n}, x\right)} \mathrm{d} x \\
\quad= & \liminf _{t \rightarrow \infty}\left(\int_{S\left(t_{0}\right)} H(t, x) \mathrm{d} S\right)^{-1} \int_{\Omega_{0, t}\left(t_{0}, t\right)} \frac{\|\boldsymbol{h}(t, x)\|^{p} \rho(x)}{p^{p} H^{p-1}(t, x)} \mathrm{d} x .
\end{aligned}
$$

Now suppose by contradiction that (3.7) fails. As in the proof of Lemma 3.1 and using (3.20) we conclude (3.16). Using the same procedure as in Lemma 3.1 we obtain (3.17) and (3.18), which contradicts to (3.21). Hence (3.7) holds.

Proof of Theorem 2.4. The proof is almost the same as the proof of Theorem 2.3. Lemma 3.2 is applied instead of Lemma 3.1.

\section{REFERENCES}

[1] M. Atakarryev, A. Toraev, Oscillation and non-oscillation criteria of Knezer type for elliptic nondivergent equations in unlimited areas, Proc. Acad. Sci. Turkmen. SSR 6 (1986), 3-10 (russian).

[2] J. I. Díaz, Nonlinear Partial Differential Equations and Free Boundaries, Vol. I Elliptic Equations, Pitman Publ., London 1985.

[3] O. Došlý, Methods of oscillation theory of half-linear second order differential equations, Czech. Math. J. 125, 2 (2000), 657-671.

[4] O. Došlý, Oscillation criteria for half-linear second order differential equations, Hiroshima Math. J. 28 (1998), 507-521.

[5] O. Došlý, A. Lomtatidze, Oscillation and nonoscillation criteria for half-linear second order differential equations, submitted.

[6] O. Došłý, R. Mařík, Nonexistence of positive solutions for PDE's with $p$-Laplacian, Acta Math. Hungar., 90 (1-2) (2001), 89-107.

[7] F. Fiedler, Oscillation criteria of Nehari-type for Sturm-Liouville operators and elliptic operators of second order and the lower spectrum, Proc. of Roy. Soc. of Edinb. 109A (1988), $127-144$.

[8] J. Jaroš, T. Kusano, N. Yoshida, A Picone-type identity and Sturmian comparison and oscillation theorems for a class of half-linear partial differential equations of second order, Nonlin. Anal. TMA 40 (2000), 381-395.

[9] N. Kandelaki, A. Lomtatidze, D. Ugulava, On the oscillation and nonoscillation of a second order half-linear equation, Georgian Math. J.7 (2) (2000), 347-353. 
[10] Q. Kong, Interval criteria for oscillation of second-order linear ordinary differential equations, J. Math. Anal. Appl. 229 (1999), 258-270.

[11] T. Kusano, Y. Naito, Oscillation and nonoscillation criteria for second order quasilinear differential equations, Acta. Math. Hungar. 76 (1997), 81-99.

[12] T. Kusano, Y. Naito, A. Ogata, Strong oscillation and nonoscillation of quasilinear differential equations of second order, Diff. Equations Dyn. Syst. 2 (1994), 1-10.

[13] H. J. Li, Oscillation criteria for half-linear second order differential equations, Hiroshima Math. J. 25 (1995), 571-583.

[14] R. Mařík, Hartman-Wintner type theorem for PDE with $p$-Laplacian, EJQTDE 18 (2000), Proc. 6th. Coll. QTDE, p.1-7.

[15] R. Mařík, Oscillation criteria for PDE with $p$-Laplacian via the Riccati technique, J. Math. Anal. Appl. 248 (2000), 290-308.

[16] W. F. Moss, J. Piepenbrick, Positive solutions of elliptic equations, Pacific J. Math. 75 No. 1 (1978), 219-226

[17] E. Müller-Pfeiffer, Oscillation criteria of Nehari-type for the Schödinger equation, Math. Nachr. 96 (1980), 185-194.

[18] M. Naito, Y. Naito, H. Usami, Oscillation theory for semilinear ellitpic equations with arbitrary nonlinearities, Funkcial. Evkac. 40 (1997), 41-553

[19] E. W. Noussair, C. A. Swanson, Positive solutions of semilinear Schrödinger equations in exterior domains, Ind. Uni. Math. J. 6 (1979), 993-1003.

[20] E. W. Noussair, C. A. Swanson, Oscillation of semilinear elliptic inequalities by Riccati equation, Can. Math. J. 22 No. 4 (1980), 908-923.

[21] Ch. G. Philos, Oscillation theorems for linear differential equations of second order, Arch. Math (Basel) 53 (1989), 483-492.

[22] U.-W. Schminke, The lower spectrum of Schrödinger operators, Arch. Rational Mech. Anal. 75 (1989), 147-155.

[23] A. Toraev, Ob oscilacii resenij ellipticeskich uravnenij, Dokl. Akad. Nauk SSSR 280 (1985), 300-303.

[24] Q.R. Wang, Oscillation and asymptotics for second-order half-linear differential equations, Appl. Math. Comp. 122 (2001), 253-266. 